\title{
INFLUENCE ON THERMAL BARRIER COATING DELAMINATION BEHAVIOUR OF EDGE GEOMETRY
}

\author{
H Brodin ${ }^{1,2} \mathrm{X}-\mathrm{H} \mathrm{Li}^{2}$, S Sjöström ${ }^{1,2}$ \\ 1) Department of Mechanical Engieering, Linköping University, SE-581 83 LINKÖPING, Sweden \\ 2) SIEMENS Industrial Turbomachinery AB, SE-612 83 FINSPONG, Sweden
}

\begin{abstract}
Ceramic thermal barrier coatings are commonly used in gas turbine hot components (e.g., combustor liners/buckets and guide vane platforms). In components that are only partially coated or have cooling-air outlets, coating-end stress singularities may lead to the spallation of the coating. Depending on the geometry of the transition from coated to uncoated material, the severity of the stress singularity will vary. One way of decreasing the severity of the stress singularity is by introducing a chamfer angle $\phi<90^{\circ}$ at the coating end. In the present study, a thin thermal barrier coating system has been studied. Bondand top coats have been sprayed to a thickness of $150 \mu \mathrm{m}$ and $350 \mu \mathrm{m}$, respectively. Vacuum-plasma-spraying technology was used, and the test specimens were rectangular $(30 \times 50 \times 5 \mathrm{~mm})$ coupons of a nickel-based superalloy, Haynes 230. A NiCrAISiY bond coat and an $\mathrm{Y}_{2} \mathrm{O}_{3}$ partially stabilised $\mathrm{ZrO}_{2}$ top coat were used. In order to achieve well-defined chamfers, sprayed coupons were ground on the edges with $\mathrm{SiC}$ grinding paper to desired geometry. By inspections of cross-sections that had not undergone thermal fatigue cycling, it was ensured that no damage was introduced into the system.

Mechanical testing was done in a thermal cyclic test rig where specimens are heated in a furnace and cooled with compressed air. FE modelling of the system has been done, aiming to support the findings from thermal fatigue tests. A parametric study including variation of the chamfer angle $\phi$ has been made and the stress state near the chamfer evaluated. Evaluation of fatigue damage can be done visually for observation of coating failure (macroscopic observation on coating surface). $20 \%$ area with complete spallation was considered as thermal barrier coating failure. For evaluation of damage development, additional light microscopy investigations of cross-sections have been carried out.
\end{abstract}

Results show that the fatigue life benefits from introduction of a chamfer angle at the coating end during thermal fatigue cycling.

Keywords: thermal barrier coating, thermal fatigue, modelling, crack initiaton, stress singularity

\section{Introduction}

A thermal barrier coating is built up of (at least) two layers. Facing the environment is a ceramic top coat (TC), designed to meet requirements on low thermal conductivity. Below the TC is a metallic bond coat, providing good adherence for the top coat through surface roughness and formation of dense and adherent oxides. Thermal barrier coatings are mainly used on stationary and rotating hot components in land-based and airborne gas turbines. Figure 1 shows a schematic picture of a 
thermal barrier coating system. In the figure is also introduced the thermally grown oxide (TGO) that forms at high temperature. Primarily the oxide is alumina formed by oxidation of the aluminium-rich BC [1].

In the design of a spallation fatigue life model several factors, such as thermal/mechanical loads, multiaxiality, oxidation, ageing and other material-related aspects need to be considered. Several proposals have previously been presented in the literature. Models based on degradation during high temperature exposure (i.e. diffusion, oxidation and corrosion-related phenomena) have been proposed by Whittle et.al. [2], Sisson et.al. [3, 4] and Strangman et.al. [5]. A Coffin-Manson approach is used in the so-called NASA-model, first developed by Miller [6] and further refined by DeMasi et.al. [7] and Cruse et.al. [8]. Another strain-based model was presented by Pilsner et.al. [9], where oxidation and edge effects were taken into account. Stress-life approaches have been suggested as well. Liu et.al. [10] and Liu [11] have presented a life prediction model based on in-plane stresses (the Basquin equation). An energy release rate-approach has been suggested by He et.al. [12, 13]. The present work aims to add further knowledge to a fracture mechanics based model proposed by Jinnestrand and Brodin [1, 14]. The model is briefly described below and is founded through a physically sound mathematical model supported in experimental findings from mechanical/thermomechanical testing.

Common for all these methods is the lack of how to treat the stress-state at the transition from coated to uncoated material, where a stress-concentration is expected [15]. One way of reducing the stress at the coating end is by introduction of a chamfer on the coating edge. The aim of the present work is to compare measurements of fatigue life with FE- and analytical results of the stress-state at a chamfered edge and to couple the local conditions to existing life prediction tools.

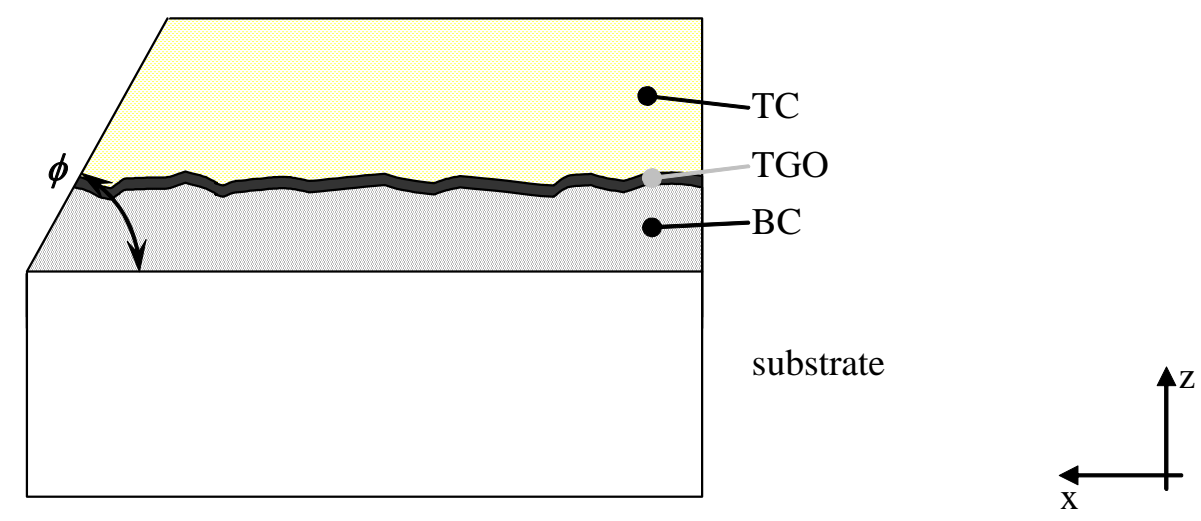

Fig. 1. Definition of chamfer angle $\phi$ produced by grinding.

\section{TBC delamination and spallation}

On planar surfaces with small curvature spallation can occur due to growth of delamination cracks at or near the TC/BC interface. With long dwell time at high temperature, out-of-plane (normal) stresses at the interface are introduced and turn tensile upon cooling [16]. As a result, cracks can grow either in the thermally grown oxide at the TC/BC interface or in the TC near the TC/TGO interface [17]. Evans et.al. have proposed a number of factors that influence TBC spallation behaviour [18]. These are: TGO in-plane compressive and out-of-plane tensile loading, imperfections at the TC/BC interface and large scale buckling. Thermal stresses at the TC/BC interface are introduced due to TGO growth during operation, interface geometry (i.e. thermal cycling) and thermal mismatch between ceramic TC and metallic BC. During thermal cycling cracks are prone to form at the metal/ceramic interface. During continued cyclic thermal loading these cracks will grow and link up to larger cracks. Finally the cracks will flake off due to buckling as discussed in literature [19-22]. The schematic process of TBC degradation is shown in Figure 2, from [1]. 


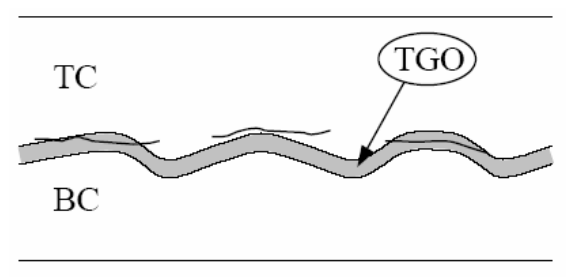

(a) $\mathrm{N}<<\mathrm{N}_{\mathrm{f}}$

Microcracking in TGO and TC

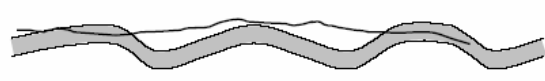

(b) $\mathrm{N} \rightarrow \mathrm{N}_{\mathrm{f}}, \mathrm{T}=\mathrm{T}_{\mathrm{HIGH}}$

Coalescence of cracks in previous cycles

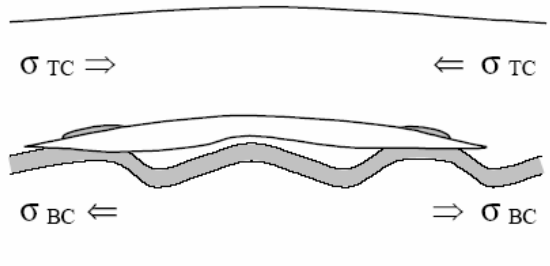

(c) $\mathrm{N} \rightarrow \mathrm{N}_{\mathrm{f}}, \mathrm{T} \rightarrow \mathrm{T}_{\text {Low }}$

Buckling, driven by delaminations

(due to $\alpha_{\mathrm{TC}}<\alpha_{\mathrm{BC}}<\alpha_{\mathrm{SUB}}$ )

Fig. 2. Damage development in thermal barrier coatings, generalised behaviour, a) formation of small interface cracks at or near the interface between top-and bond coat, b) coalescence of delaminations and c) large scale delamination (spallation).

Damage development in TBC systems can be described as the area fraction of the TC/BC interface that has spalled off in relation to the total interface area. Formation and growth of cracks can at any time increment be described as a summation of all present cracks that will contribute to spallation. The problem has previously been treated in literature by Brodin and Li [17] and Brodin [23]. In the most general description, the damage parameter $D$ can be measured on a cross section and then be deduced from measurement of length measures rather than measurements of area according to:

$$
D=\frac{\sum_{i} l_{i}^{\mathrm{TGO}}+\sum_{j} l_{j}^{\mathrm{TGO} / \mathrm{TC}}+\sum_{k} l_{k}^{\mathrm{TC}}}{L}
$$

where $I_{i}{ }^{\mathrm{TGO}}$ are individual interface cracks $i, I_{j}{ }^{\mathrm{TGO} / \mathrm{TC}}$ are individual kink cracks $j$ that initiate at the interface and propagate in the top coat and $I_{k}^{\mathrm{TC}}$ are individual top coat cracks $k . L$ is total the measured (investigated) length of interface. The measure $D$ is used below in the mathematical description of TBC damage development on a small scale (over an interface asperity). From visual inspections of fatigue-tested material systems a comparison can be made between measured delamination data and predicted values. The measurement is made on polished cross-sections of TC/BC/substrate.

\section{Material system}

The material system used is a thin TBC system with top- and bond coat thicknesses of $200 \mu \mathrm{m}$ and $350 \mu \mathrm{m}$ respectively. The top coat is yttria partially stabilized zirconia prepared by air plasma spray (APS) technique and the bond coat is a NiCrAISiY alloy produced with the vacuum plasma spray (VPS) method.

Rectangular coupons $(50 \times 30 \times 5 \mathrm{~mm})$ of the nickel-based superalloy Haynes 230 were coated to desired TBC thickness. Compositions of substrate, BC and TC are presented in Table 1. 
Table 1. Alloy compositions for substrate, bond coat and top coat (in wt\%).

\begin{tabular}{cccccccccccccccccc}
\hline & Ni & Cr & W & Mo & Fe & Co & Mn & Si & Al & C & La & B & Ta & Y & $\mathbf{Z r O}_{2}$ & $\mathbf{Y}_{2} \mathbf{O}_{3}$ \\
\hline substrate & bal. & 22 & 14 & 2 & $\leq 3$ & $\leq 5$ & 0,5 & 0,4 & 0,3 & 0,1 & 0,02 & $\leq 0,015$ & - & - & - & - \\
BC & bal. & 25 & - & - & - & - & - & 2,6 & 5 & - & - & - & 1 & 0,6 & - & - \\
TC & - & - & - & - & - & - & - & - & - & - & - & - & - & - & bal. & 7 \\
\hline
\end{tabular}

After coating production the edges were modified to desired geometry with a well-defined coating chamfer angle $\phi$, according to Figure 1. The chamfered edge was produced by grinding to desired shape with silicon carbide wet grinding paper. A grinding guide block with corresponding chamfer angle was used to ensure correct chamfer geometry of the TBC. In the present work the chamfer angle was chosen to $\phi=90^{\circ}, 75^{\circ}$ and $60^{\circ}$.

The coating system used is shown in Figure 3.

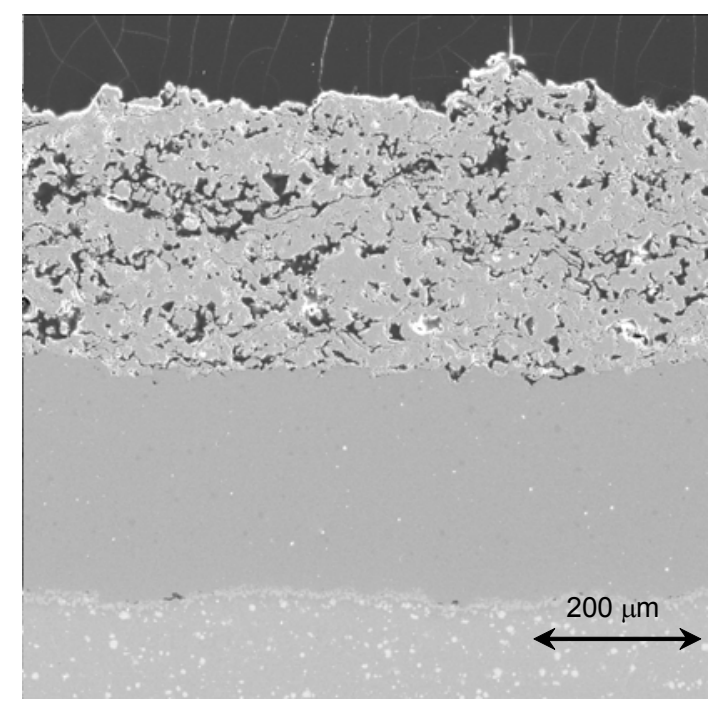

Fig. 3. TBC system used in present study.

\section{Experimental procedure}

Fatigue testing of chamfered and as-received material system (superalloy with TBC) has been performed as thermal cyclic fatigue tests (TCF). Tests are conducted in air with heating in a furnace followed by cooling in air to low temperature. In the current experiment, the thermal cycle spanned between $T_{\min }=100^{\circ} \mathrm{C}$ and $T_{\max }=1100^{\circ} \mathrm{C}$. The cycle time was $4200 \mathrm{~s}$ with a dwell time of 2400 s at the maximum temperature. The tests were monitored by a video camera for determination of number of cycles to spallation. Spallation was defined as $20 \%$ reduction of interface area adherence and measured visually after fatigue cycling from a video camera recording.

\section{Fatigue life modelling}

The model previously presented is based on fracture-mechanical modelling and corresponding material testing described later on. From an analysis of the stress state at the crack tip, crack growth is described by an expansion of the classic Paris law equation,

$$
\frac{d a}{d N}=C\left(\Delta K_{I}\right)^{n}
$$

A micromechanical model has been made of the TBC aggregate substrate/BC /TGO /TC. The model has been subjected to the following thermal and mechanical load cycles: 
i) A thermal cycle with thermal boundary conditions representative for industrial gas turbine components.

ii) A case with the same thermal cycle as in i) but with the addition of an out-of-phase external load, corresponding to a case typical for a hot-spot area.

At or near the BC/TGO/TC interfaces, cracks will be initiated and propagated as sketched in Figure 4 . The model has been FE-analysed for different interface geometries through variations of the $\mathrm{H} / \mathrm{L}$ ratios, different crack lengths and TGO thicknesses. Also, the effect of variation in crack pattern (failure mode) is analysed. In this way, we have been able to set up a database of FE-computed stress intensities $K_{I}$ and $K_{I I}$ and energy release rate $G$ for these variations.

The fatigue crack analysis in this interface, or, rather, interface-near crack case differs from that in a homogeneous material by the fact that growth can continue under sustained mode I/mode II mixity. In Paris law, we need to take this new behaviour into account by introducing an equivalent stress intensity factor range, $\Delta K_{e q}$. Alternatively, the description of the crack driving force is done by the range $\Delta G$ of the energy release rate, with the mode mixity taken into consideration through a factor $\lambda$.

$$
\frac{d a}{d N}=C\left(\Delta K_{e q}\right)^{n}=C_{2}(\lambda \cdot \Delta G)^{n_{2}}
$$

where $\lambda$ can, for instance, be defined in terms of the mode mixity $\varphi=\arctan \left(K_{I I} / K_{I}\right)$ introduced by Hutchinson and Suo [24]:

$$
\lambda=1-\left(1-\lambda_{0}\right)\left[\frac{2}{\pi} \arctan \left(\frac{K_{I I}}{K_{I}}\right)\right]^{m}
$$

Note that $K_{I I}=0 \Rightarrow \lambda=1$ and $K_{I}=0 \Rightarrow \lambda=\lambda_{0}$ (where $\lambda_{0}<1$, since it is more difficult to drive a crack forward under a pure mode II load).

Normally, Paris law is used for crack growth estimations. However, since growth of the FE-analysed 'prototype' crack (together with all the neighbouring cracks) can be identified as growth of a general damage measure $D$ according to the previous discussion and Eq. (1), we now write Eq. (3) as a damage growth equation instead:

$$
\frac{d D}{d N}=C_{2}(\lambda \cdot \Delta G)^{n_{2}}
$$

with the same definition of $\lambda$ as in Eq. (4). After proper identification of $C_{2}, n_{2}, \lambda_{0}$ and $m$ from testing, one can therefore integrate Eq. (5) to compute $D=D(N)$. Complete failure is expected when the damage $D$ is equal to unity.

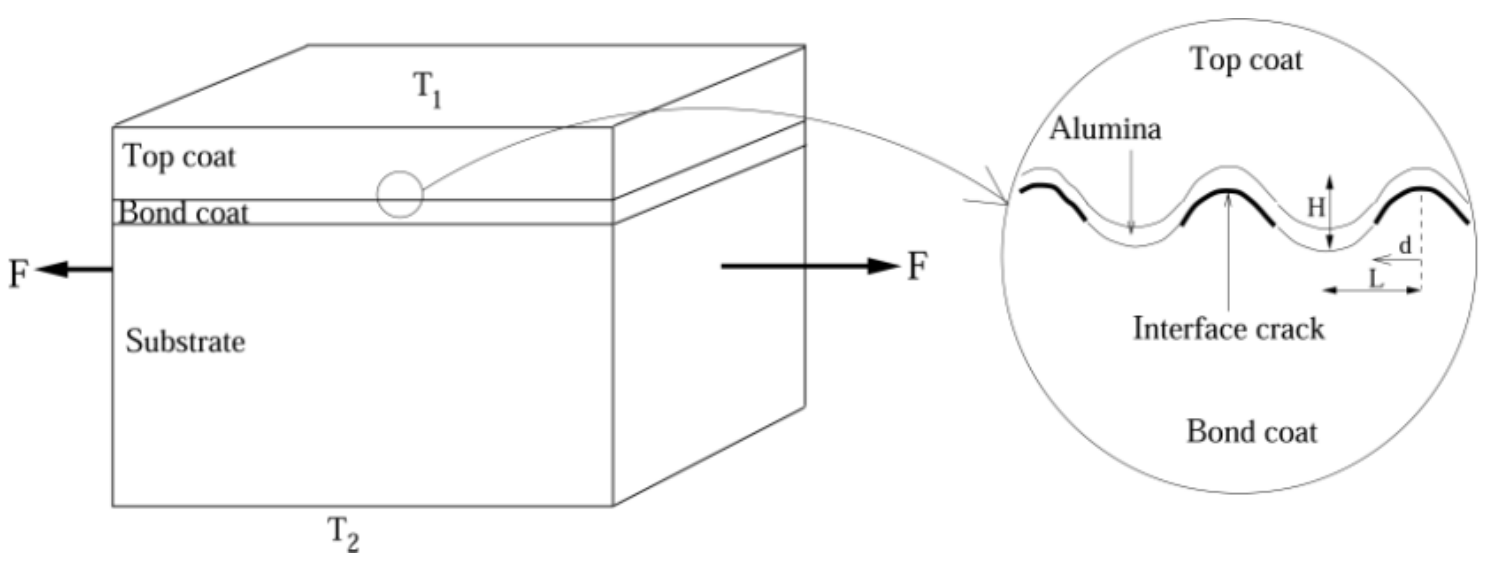

Fig. 4. Geometry considered for FE-analysis of TBC spallation behaviour. 


\section{Edges}

Experimental experience shows that there is a clear tendency of the interface cracks starting from the edges of the test specimen, where there is a stress singularity. It is well known from, for instance from the work by Bogy [25, 26, 27], that the stress state at the edge of a biomaterial aggregate depends only on the two composite parameters

$$
\begin{aligned}
& \alpha=\frac{\mu^{(2)} m^{(1)}-\mu^{(1)} m^{(2)}}{\mu^{(2)} m^{(1)}+\mu^{(1)} m^{(2)}} \text { and } \\
& \beta=\frac{\mu^{(2)}\left[m^{(1)}-2\right]-\mu^{(1)}\left[m^{(2)}-2\right]}{\mu^{(2)} m^{(1)}+\mu^{(1)} m^{(2)}},
\end{aligned}
$$

first given by Dundurs [28]. In Eqs. (6) and (7), $\mu^{(.)}$is the shear modulus,

$$
m^{(.)}=\left\{\begin{array}{l}
\frac{4}{1+v} \text { for P.S } \\
4(1-v) \text { for P.D. }
\end{array}\right.
$$

and the superscripts ${ }^{(1)}$ and ${ }^{(2)}$ refer to the upper and the lower, respectively, of the two materials.

To solve the stress state near the bimaterial interface, the Mellin transform

$$
F_{M}(s)=\int_{0}^{\infty} f(x) x^{s-1} d x
$$

has often been used. The Laplace equation governing the solution of the Mellin transform of Airy's function can then be written

$$
\left(\frac{\partial^{2}}{\partial \theta^{2}}+s^{2}\right)\left[\frac{\partial^{2}}{\partial \theta^{2}}+(s+2)^{2}\right] \hat{\Phi}^{(k)}=0 ; k=1,2 .
$$

where $\hat{\Phi}$ is the Mellin transform of Airy's stress function $\Phi$.Eq. (10) has the solution

$$
\hat{\Phi}^{(k)}=a^{(k)}(s) \sin (s \theta)+b^{(k)}(s) \cos (s \theta)+c^{(k)}(s) \sin [(s+2) \theta]+d^{(k)}(s) \cos [(s+2) \theta] ; k=1,2,
$$

where $a^{(k)}(s), \ldots ; k=1,2$ are 8 unknown complex-valued functions of the complex variable $s=p+i q$, which must bechosen so that boundary and interface continuity conditions are fulfilled. See Figure 5 for definitions of $r$ and $\theta$.

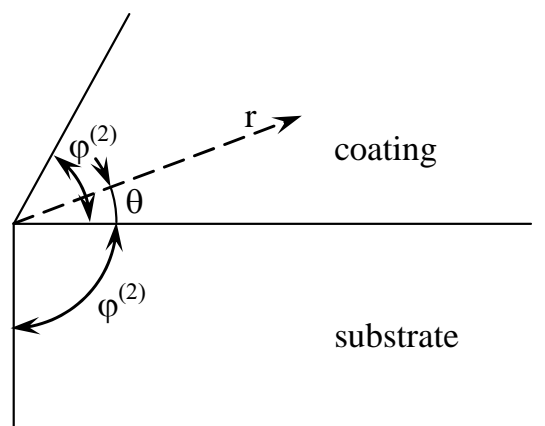

Fig. 5. Coating edge geometry. 
The solution (11) has a number of poles $s_{n}=p_{n}+i q_{n}$. If there is at least one pole such that $-2<\operatorname{Re}\left(s_{n}\right)<-1$, then the one of these (namely, $s_{1}=p_{1}+i q_{1}$ ) that has the largest real part gives rise to a stress singularity for $r \rightarrow 0$, since in this case

$$
\lim _{r \rightarrow 0} \sigma_{i j}^{(k)}(r, \theta)=\left\{\begin{array}{l}
2 r^{-\left(p_{1}+2\right)}\left\{\operatorname{Re}\left[T_{i j}^{(k)}\left(s_{1}, \theta\right)\right] \cos \left(q_{1} \ln r\right)+\operatorname{Im}\left[T_{i j}^{(k)}\left(s_{1}, \theta\right)\right] \sin \left(q_{1} \ln r\right)\right\}+o\left[r^{\left.-\left(p_{1}+2\right)\right] ; q_{1} \neq 0}\right. \\
r^{-\left(p_{1}+2\right)} T_{i j}^{(k)}\left(s_{1}, \theta\right)+o\left[r^{-\left(p_{1}+2\right)}\right] ; q_{1}=0
\end{array}\right.
$$

In Eq. (12),

$$
T_{i j}^{(k)}\left(s_{1}, \theta\right)=\lim _{s \rightarrow s_{1}}\left(s-s_{1}\right) \hat{o}_{i j}^{(k)}(s, \theta),
$$

where $\hat{O}_{i j}^{(k)}(s, \theta)$ is the (Mellin-plane) stress solution derivable from the Airy-function solution (11). The singularity is of the order $r^{\lambda}=r^{-\left(p_{1}+2\right)}$. Note that if $q_{1} \neq 0$, then the stress solution is oscillatory, a property which is also well-known for the asymptotic stress solution near an interface crack.

In the limiting case $s_{1}=-2$, there is a stress singularity of order $\ln r$; in all other cases there is no stress singularity.

A couple of examples of results of computed singularity orders $\lambda$ for a material combination typical in a TBC are shown in Figs.6 and 7. Data used are listed in Table 2.

For $\phi^{(2)}=90^{\circ}$, the stress singularity is completely avoided for sufficiently small 'coating angles' $\phi^{(1)}$.As can be seen in Fig. 6 , $\phi^{(1)}<70^{\circ}$ will be sufficient for both $T=20^{\circ} \mathrm{C}$ and $T=800^{\circ} \mathrm{C}$.

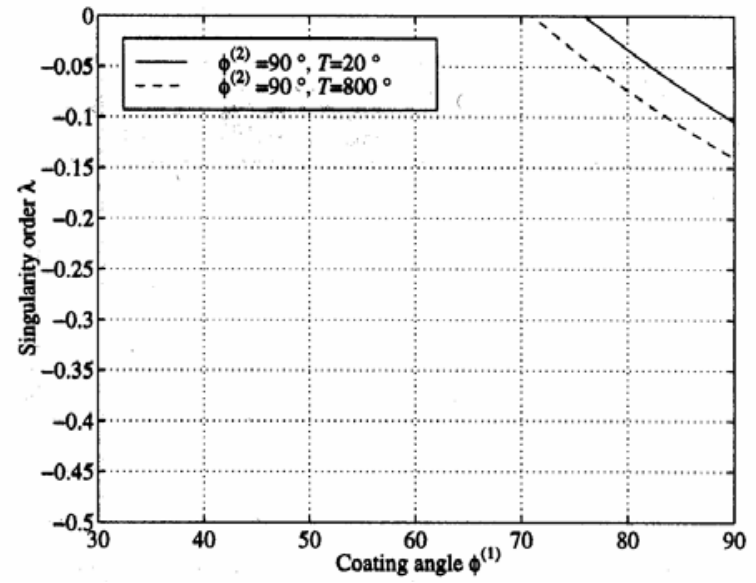

Fig. 6. Stress singularity $\lambda$ as a function of 'coating angle' $\phi^{(1)}$ for 'substrate angle' $\phi^{(2)}=90^{\circ}$. Plane deformation

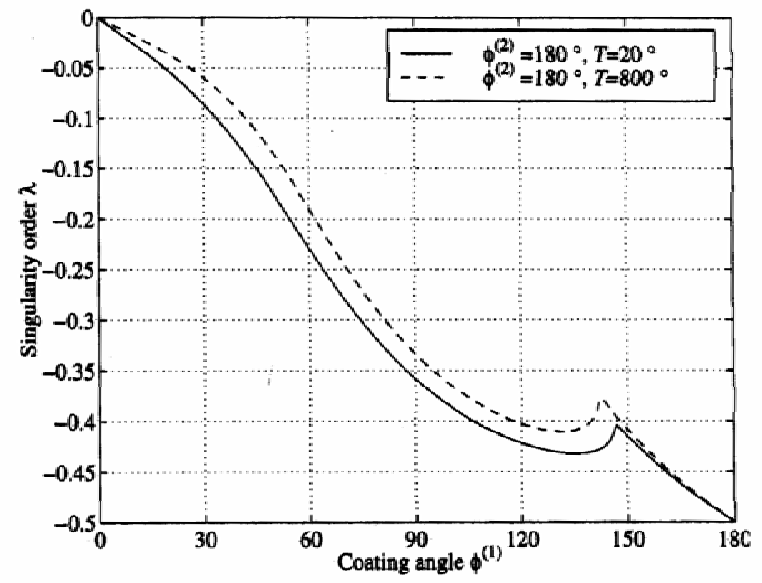

Fig. 7. Stress singularity $\lambda$ as a function of 'coating angle' $\phi^{(1)}$ for 'substrate angle' $\phi^{(2)}=180^{\circ}$. Plane deformation. 
Table 2. Data for computed example

\begin{tabular}{|c|c|c|c|c|c|c|}
\hline & $T$ & $\mu^{(k)}$ & $v^{(k)}$ & $m^{(k)}$ & $\alpha$ & $\beta$ \\
\hline Coating, $k=1$ & \multirow{2}{*}{$20^{\circ} \mathrm{C}$} & $19.2 \cdot 10^{9} \mathrm{~Pa}$ & 0.25 & 3.0 & \multirow{2}{*}{0.6234} & \multirow{2}{*}{0.2015} \\
\hline Substrate, $k=2$ & & $87.2 \cdot 10^{9} \mathrm{~Pa}$ & 0.21 & 3.16 & & \\
\hline Coating, $k=1$ & \multirow{2}{*}{$800^{\circ} \mathrm{C}$} & $10.0 \cdot 10^{9} \mathrm{~Pa}$ & 0.25 & 3.0 & \multirow{2}{*}{0.7310} & \multirow{2}{*}{0.2391} \\
\hline Substrate, $k=2$ & & $67.8 \cdot 10^{9} \mathrm{~Pa}$ & 0.21 & 3.16 & & \\
\hline
\end{tabular}

\section{Fatigue of chamfered TBC}

Results have been obtained for TCF tests of chamfered TBC systems. The results are presented in Figure 8 . It is obvious that a chamfer has a beneficial influence on fatigue life. A smaller coating chamfer angle $\phi$ gives a longer TBC spallation fatigue life.

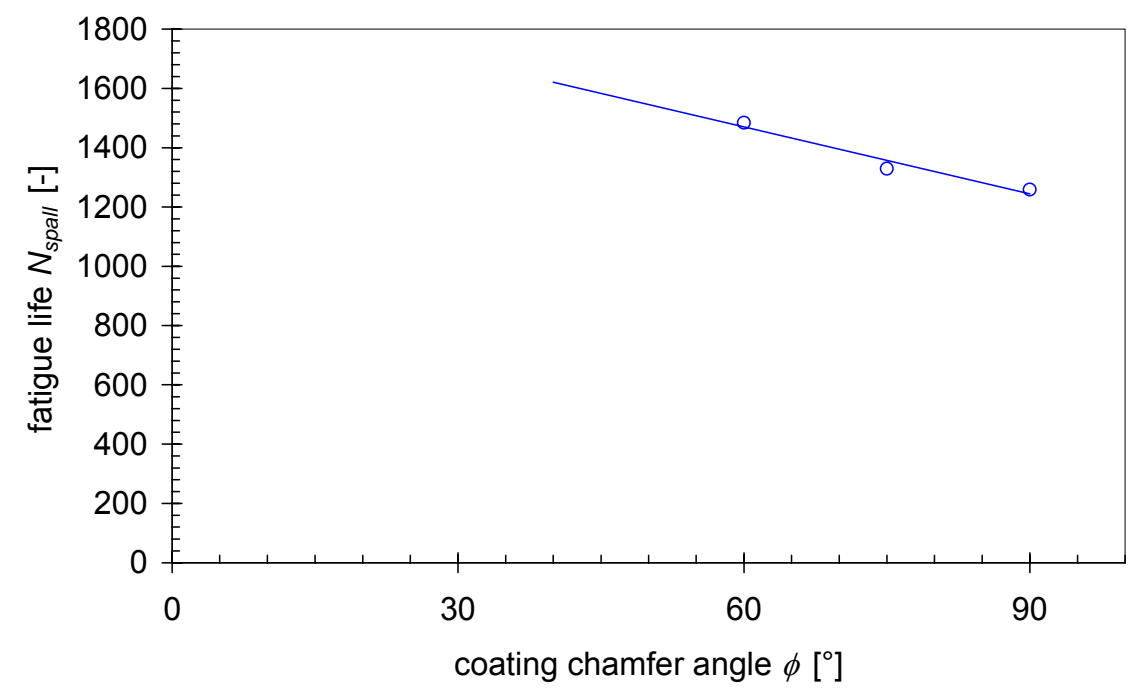

Fig. 8. TCF results of a chamfered TBC system. Graph shows spallation fatigue life $N_{\text {spall }}$ as a function of coating chamfer angle $\phi$. Data are average values, two samples per measurement point.

A linear elastic FE model has been evaluated for a number of coating chamfer angles $\phi$ to see the extent of a stress concentration near the free edge. Results are presented for TBC modelled with an interface without asperities. Therefore local effects of tensile out-of-plane stresses are ignored in the analysis. The results are presented as stress profiles for a crack free TBC system. Results for in-plane $\left(\sigma_{\mathrm{x}}\right)$ and out-of-plane $\left(\sigma_{\mathrm{z}}\right)$ stress components are shown in Figure 9 and 10. 


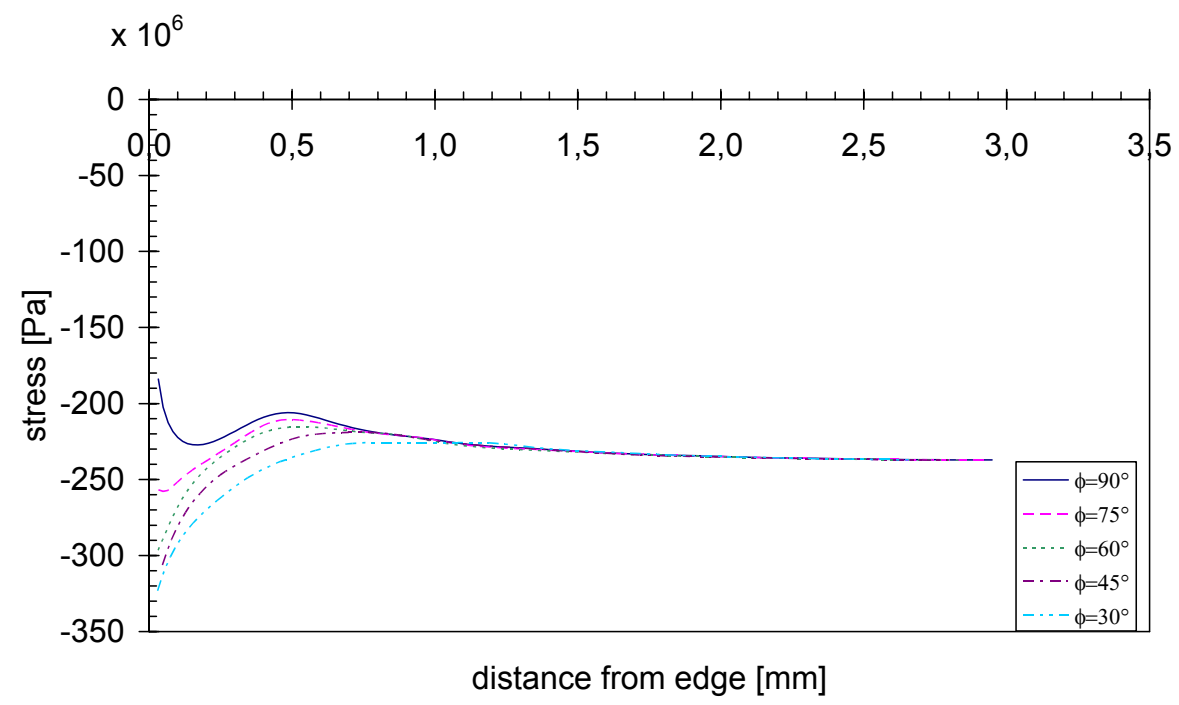

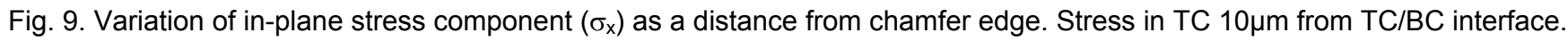
For definition of directions, see Fig. 1.

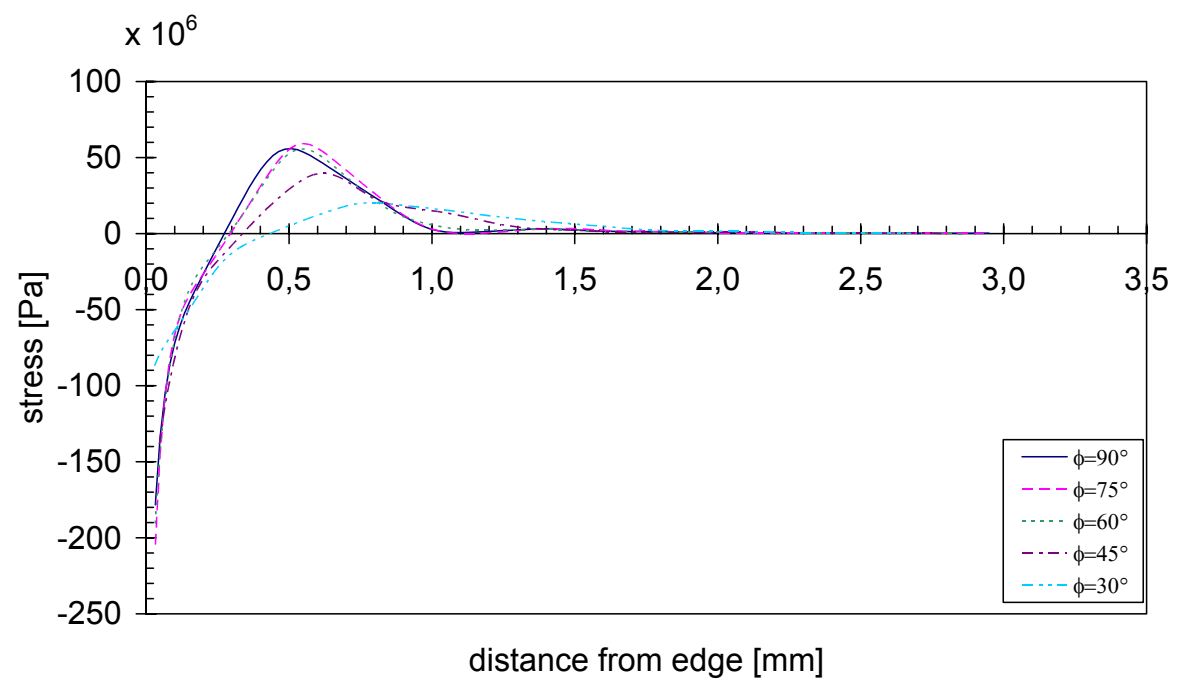

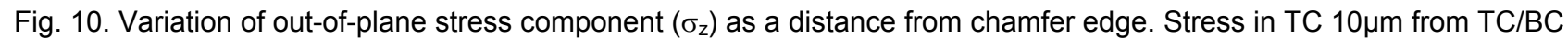
interface. For definition of directions, see Fig. 1.

From Figure 8 and 9 it is clear that influence on the stress state from the edge are present for any given coating chamfer edge angle $\phi$, but the influence diminishes rapidly with increased in-plane distance from the chamfered edge. In a position $2 \mathrm{~mm}$ away from the edge, the stress field is equal in all cases investigated.

\section{Discussion}

The analytical investigation performed indicates that the stress singularity at the TBC edge decreases with decreased TC chamfer angle $\phi$ as shown in Figure 4. A lower chamfer angle will not cause a stress singularity. TCF test results indicate that a decrease from $\phi=90^{\circ}$ to $\phi=60^{\circ}$ will not give dramatic changes to the expected fatigue life. Instead a gradual improvement of fatigue performance is expected with decreased chamfer angle $\phi$. This is logical if two conditions are assumed: 
- If Figure 9 and 10 are considered, the TBC loading is dependent on geometry only near the chamfer edge. The stress-field is different in an area extending only 2 millimetres into the bulk of the TBC system. In the interior of the TBC the stress-field is independent of macroscopic (chamfer) geometry. It is reasonable to state that the edge gives a local influence on cracking behaviour, i.e. in early stages of crack growth.

- $\quad$ TBC crack propagation internally in the material system is independent on local effects during crack initiation. In this region crack growth can be predicted with existing TBC lifing tools, such as the one presented above, Eqs. (3) - (5) with a number of cracks summing up to a damage level that is judge to be detrimental from material integrity point of view as suggested in Eq. (1).

With these assumptions made, the gradual improvement of TBC performance with chamfer angle $\phi$ can be explained. The stress singularity at the chamfer edge will influence crack initiation due to the (in comparison) severe stress state for high chamfer angles ( $\phi>70^{\circ}$ gives a stress singularity). For short crack growth the behaviour will be different for high or low chamfer angles. After crack growth into the bulk the stress field around the crack tip is equal for all geometries. All cracks have travelled away from the area where differences in stress state are expected and crack propagation is only governed by the stress state within the TBC system caused by interface asperities and thermal expansion mismatch.

A way forward for incorporating edge effects in a life prediction tool would therefore be to use a model according to Eq. (5) where the stress field at free edges is allowed to influence crack initiation and early crack growth behaviour through a locally altered stress intensity (and energy release rate). After crack travel through the region with influence on stress state from geometry the stress field changes and the crack growth is governed by "normal" conditions in the material system.

\section{Conclusions}

An analysis of the stress state at a TBC edge (transition from coated to uncoated material) has been evaluated analytically and experimentally and also supported by an FE simulation. The results indicate that it is beneficial to introduce a tapering at the transition from coated to uncoated material. The tapering is done here as a chamfer angle at the edge of small test coupons. The results can be summarised as:

- For steep chamfer angles $\left(>70^{\circ}\right)$ a stress singularity can be shown by an analytical analysis.

- Experimental investigations in terms of thermal cyclic testing of thermal barrier coating system gives evidence of the benefit of tapering; a gradual increase of spallation fatigue life with decreasing coating chamfer angle is shown.

- A linear elastic FE analysis of a TBC aggregate without interface asperities shows that the stress field is altered near the edge. However, only a distance from the edge up to $2 \mathrm{~mm}$ shows influence of the stress caused by the edge.

\section{Acknowledgments}

The authors would like to acknowledge The Swedish National Energy Administration, project KME 106 / 706 for financial support.

\section{References}

[1] Brodin, H., "Failure of thermal barrier coatings under thermal and mechanical fatigue loading" Linköping University, Linköping Studies in Science and Technology, Dissertation No 898, October 2004.

[2] Whittle, D.P., Evans, D.J., Scully, D.B. and Wood, G.C., "Compositional changes in the underlying alloy during the protective oxidation of alloys", Acta Metall, 15, 14121-1430 (1967).

[3] Lee, E.Y., Biederman, R.R. and Sisson, R.D., In: Proceedings of the DOE - 1990 Coatings for advanced heat engines workshop, Castine, Maine, August 6-9, 293-315 (1990).

[4] Lee, E.Y., Biederman, R.R. and Sisson, R.D., "Development of reaction products at the interface between partially stabilized zirconia and the MCrAlY bond coat in thermal barrier coatings" Mémories et Études Scientifiques Revue de Metallurgie, 88, Mai, 307-311 (1991).

[5] Strangman, T.E., Neumann, J. and Liu, A., "Thermal Barrier Coating Life Prediction Model Development", Garret Engine Company, NASA CR-179507, (1986).

[6] Miller, R.A.," Oxidation-based model for thermal barrier coating life", J. Am. Ceram. Soc., 67, 517-521 (1984). 
[7] DeMasi, J.T., Manning, S.L., Oritz, M. and Sheffler, K.D., "Thermal barrier coating life prediction model development Phase I“, NASA CR-182230, (1989).

[8] Cruse, T.A., Stewart, S.E. and Oritz, M., J. Eng. Gas Turbines Power, 110, 610-616 (1998).

[9] Pilsner, B., Hillery, R., McKnight, R., Cook, T. and Hartle, M., "Thermal barrier coating life prediction model", Surf. Coat. Technol., 32, 305-306 (1987).

[10] Liu, Y., Persson, C., Gualco, T., Haubold, D., Schwingel, D., Taylor, R. and Wigren, J., "Life prediction of thermally cycled thermal barrier coatings", In Proceedings of the seventh fatigue conference, Beijing, P.R. China, Higher Education Press, 1927-1932 (1999).

[11] Liu, Y., "Life predictions and mechanical behavior of thermal barrier coatings", Lund University, Department of Mechanical engineering, May 2003.

[12] He, M.Y, Hutchinson, J.W. and Evans, A.G., "Simulation of stresses and delamination in a plasma-sprayed thermal barrier system upon thermal cycling", Mater. Sci. Eng. A, 345, 172-178 (2003).

[13] He, M.Y, Hutchinson, J.W. and Evans A.G., "Large deformation simulations of cyclic displacement instabilities in thermal barrier systems", Acta Mater. 50, 1063-1073 (2002).

[14] Jinnestrand, M., "Delamination in APS applied thermal barrier coatings: Life modelling", Linköping University, Linköping Studies in Science and Technology, Dissertation No 902, October 2004.

[15] Sjöström S., Burman J. and Jinnestrand M., "Lifetime prediction of gas turbine components with thermal barrier Coatings", Int. J. Appl. Mech. Eng., 7, 205-220 (2002).

[16] Jinnestrand, M. and Sjöström, S., "Investigation of 3D FE simulations of delamination crack initiation in TBC caused by alumina growth ", Surf. Coat. Technol., 185, 35-39 (2000).

[17] Brodin, H. and Li, X-H., "Fatigue life prediction of a plasma sprayed thermal barrier coating system", In: Proceedings of ITSC 2004, Osaka, Japan, (2004).

[18] Evans, A.G., He, J.W. and Hutchinson J.W., "Mechanics-based scaling laws for the durability of thermal barrier coatings", Progr. Mater. Sci., 46, 249-271 (2001).

[19] Rapp, R A and Zhang, Y S., "Hot corrosion of materials: fundamental studies" JOM., 46, 47-55(1994).

[20] Leyens, C., Wright, I.G. and Pint, B.A., "Hot Corrosion of Nickel-Base Alloys by Alkali-Containing Sulfate Deposits", Mater. Sci. Forum, 369-372, 571-578 (2001).

[21] Wang, J.S. and Evans, A.G., "Effects of strain cycling on buckling, cracking and spalling of a thermally grown alumina on a nickel-based bond coat", Acta. Mater., 47, 699-710 (1999).

[22] Chai, H., Babcock, C.D., Knauss, WG., "One dimensional modeling in laminated plates by delamination buckling", Int. J. Solid. Struct., 17, 1069-1083 (1981).

[23] Brodin, H., "Initiation and growth of delamination cracks in vacuum plasma sprayed thermal barrier coatings", In: Proceedings of ISEC 2004, Orlando, FL, (2004).

[24] Hutchinson, J.W. and Suo, Z., "Mixed mode cracking in layered materials", Adv. Appl. Mech., 29, 163-189 (1987).

[25] Bogy, B.D., "Edge-bonded dissimilar orthogonal elastic wedges under normal and shear loading", J. Appl. Mech., 35, 460-466 (1968).

[26] Bogy, B.D., "On the problem of edge-bonded elastic quarter-planes loaded at the boundary", Int. J. Solids Struct., 6, $1287-1313$ (1970).

[27] Bogy, B.D., "Two edge-bonded elastic wedges of different materials and wedge angles under surface tractions", J. Appl. Mech. 38, 377-386 (1971).

[28] Dundurs J., Discussion of edge-bonded dissimilar orthogonal elastic wedges under normal and shear loading, J. Appl. Mech. 36, (1969), pp. 650-652 